\title{
Similaridade e fatores determinantes na salinidade das águas superficiais do Ceará, por técnicas multivariadas
}

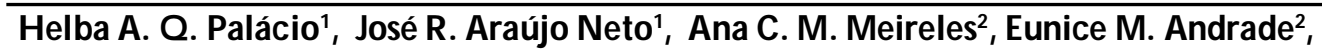 \\ Julio C. N. Santos ${ }^{2} \&$ Luiz C. G. Chaves $^{2}$
}

\begin{abstract}
RESUMO
Técnicas de estatística multivariada foram empregadas com o objetivo de identificar a similaridade dos reservatórios superficiais do Ceará, com relação à salinidade das águas e identificar os fatores determinantes da qualidade das águas. Os dados utilizados foram provenientes do banco de dados da Companhia de Gestão dos Recursos Hídricos do Ceará, no período de 1998/2009, no total de 807 amostras de 48 reservatórios. Os parâmetros considerados foram: $\mathrm{Cl}, \mathrm{Ca}^{2+}, \mathrm{M} \mathrm{g}^{2+}, \mathrm{Na}^{+}, \mathrm{HCO}_{3}$; $\mathrm{CE}$ e RAS. A análise de agrupamento hierárquico formou quatro grupos distintos, sendo determinantes os tipos e concentrações dos sais analisados. Nos grupos 1 e 2 a salinidade da água é definida principalmente pelas condições naturais de solo e clima da região e nos grupos 3 e 4, além desses fatores, a influência antrópica. 0 emprego da análise de fator/análise de componente principal promoveu a redução de sete características das águas superficiais do Estado Ceará, para dois componentes, sendo que o primeiro componente explicou $57,28 \%$ e o segundo, $26,77 \%$ da variância contida nas sete variáveis. 0 s parâmetros mais representativos na variabilidade da salinidade das águas foram $\mathrm{Cl} ; \mathrm{CE}, \mathrm{Na}^{+}$e $\mathrm{M} \mathrm{g}^{2+}$, relacionados com a solubilidade dos sais.
\end{abstract}

Palavras-chave: qualidade de água, sais, reservatórios, semiárido

\section{Similarity and determining factors in the salinity of surface waters of Ceara by mitivariate techniques}

\section{ABSTRACT}

Multivariate statistical techniques were employed in order to identify the similarity in the salinity levels of the water reservoirs of Ceará State, Brazil. Also, to identify the factors that explain the variability of water quality. The data set used in this investigation came from the Company of Water M anagement (CO GERH). The studied period extended from 1998 to 2009, in a total of 807 samples from 48 different sources. The investigated parameters were: $\mathrm{Cl}^{-}, \mathrm{Ca}^{2+}, \mathrm{M} \mathrm{g}^{2+}, \mathrm{Na}^{+}, \mathrm{HCO}_{3}$; $\mathrm{EC}$ and SAR. The $\mathrm{H}$ ierarchical Cluster Analysis identified four groups, being the types and concentrations of the analyzed salts the determining factors. In groups 1 and 2, the salinity level of the water was, mainly, due to the geology and the climate of the region. While for group 3 and 4, besides these factors, the water salinity was influenced by human activities. According to the results, the first and the second components (independent factors) explaining, 57.28 and $26.77 \%$, respectively, the total variance of seven variables. The most important parameters related to the variability of the water salinity level were $\mathrm{Cl}, \mathrm{CE}, \mathrm{N} \mathrm{a}^{+}$and $\mathrm{M} \mathrm{g}^{2+}$, which were related to salt solubility.

Key words: water quality, salts, reservoirs, semiarid

Trabalho submetido e selecionado no primeiro Simpósio Brasileiro de Salinidade realizado de 12-15/10/2010 em Fortaleza, Ceará, Brasil ${ }^{1}$ IFCE, Campus Iguatu-CE, Rodovia Iguatu/N. Alegre Km 05, S/N, Caixa Postal 38, Vila Cajazeiras, CEP 63500-000, Iguatu, CE, Fone (88) 35821000, ramal 252. E-mail: helbarauj023@yahoo.com.br; junior.bg@bol.com.br

2 DEN A/U FC, Av. Mister Hull, S/N, Caixa Postal 12.168, Campus do Pici, CEP 60455-970, Fortaleza, CE, Fone: (85) 3366-.9762. E-mail: eandrade@pq.cnpq.br; ameireles2003@yahoo.com.br; juliocesarnds@yahoo.com.br; luizcarlosguerreiro@hotmail.com 


\section{INTRODUÇÃO}

Em regiões semiáridas a escassez de água decorrente da pouca incidência de chuvas que ocorrem apenas num período de três a cinco meses por ano e são irregularmente distribuídas no tempo e no espaço, aliada à ocorrência de altas taxas de evaporação, é responsável pela intermitência de quase toda a rede hidrográfica dessas regiões (Frota Júnior et al., 2007). Este fato representa um problema severo para a captação e armazenamento desse recurso; portanto, milhares de reservatórios são construídos nessas regiões, com a finalidade principal de armazenar água para múltiplos usos, principalmente, durante os períodos de estiagem.

Embora os açudes contribuam significativamente para o abastecimento hídrico, essas regiões se deparam com um novo empasse: a qualidade das águas em muitos reservatórios não é adequada para os múltiplos usos. Essas águas, sujeitas às elevadas taxas de evaporação, tornaram-se salinas atingindo, em alguns casos, concentrações de sais que impedem seu uso para consumo humano e agricultura, principalmente no período de estiagem, quando ocorrem diminuição do escoamento e elevadas temperaturas na região (Meireles et al., 2007).

O conhecimento da composição iônica da água é primordial quando se deseja avaliar sua qualidade para fins agronômicos e, mais especificamente, para uso na irrigação (Arraes et al., 2009). A água de irrigação apresenta, na maioria das vezes, uma composição química constituída de sais de cálcio, magnésio, sódio e potássio nas formas de cloretos, sulfatos, carbonatos e bicarbonatos. Esses elementos podem apresentar diferentes proporções dependendo da fonte de água, de sua localização geográfica e da época de coleta, entre outros fatores (Silva Júnior et al., 2000). Segundo Ayers \& Westcot (1999), os principais problemas de qualidade de água para irrigação estão relacionados com a salinidade das águas, pois o excesso de sais solúveis no solo reduz a disponibilidade da água para as plantas, além da sodicidade, que pode causar sérios problemas de impermeabilização nos solos e a toxidez de alguns íons específicos.

Para se conhecer a qualidade real da água de um corpo hídrico, é imprescindível a realização de um monitoramento da qualidade da água (Girão et al., 2007). Este é um dos principais instrumentos na sustentação de uma política de planejamento e gestão de recursos hídricos. Ele funciona como um sensor que possibilita o acompanhamento do processo de uso dos cursos hídricos, apresentando seus efeitos sobre as características qualitativas das águas, visando subsidiar as ações de controle ambiental (Lemos et al., 2010), no entanto, a realização de um monitoramento envolve muitas variáveis e gera um grande número de dados, e esta situação é mais agravada quando se dá em uma escala espacial maior, em que um grande número de reservatórios é monitorado, tornandose de difícil interpretação.

Para interpretar este grande conjunto de dados, técnicas de estatística multivariada como Análise de Agrupamento Hierárquico (AAH) e Análise Fatorial/Análise de Componentes Principais (ACP) vêm sendo largamente utilizadas em dados de monitoramento de qualidade de água (Souza \& Tundisi, 2000; Helena et al., 2000; Silva \& Sacomani, 2001; Toledo \&
Nicolella, 2002; Palácio, 2004; Mendiguchía et al., 2004; Andrade et al., 2007a, 2007b; Girão et al., 2007; Palácio et al., 2009). Este tipo de análise reduz os dados de observação e permite a interpretação de diversos constituintes individualmente, uma vez que indica associações entre amostras e/ou variáveis e, ainda, possibilita identificar os possíveis fatores/fontes que influenciam o sistema de água.

Ante o exposto, aplicaram-se técnicas de estatística multivariada, Análise de Agrupamento Hierárquico (AAH) e Análise Fatorial/Análise de Componentes Principais (ACP), com o objetivo de identificar a similaridade das águas dos reservatórios superficiais do Estado do Ceará, e determinar os elementos mais significativos na variabilidade da salinidade.

\section{Material E MÉTODOS}

O estudo foi realizado no Estado do Ceará, localizado entre as latitudes $2^{\circ} 30^{\prime} 00^{\prime \prime}$ e $8^{\circ} 52^{\prime} 00^{\prime \prime} \mathrm{S}$ e as longitudes $37^{\circ} 14^{\prime} 00^{\prime \prime}$ e $41^{\circ} 30^{\prime} 00^{\prime}$ ' W. A Figura 1 ilustra a área do levantamento, destacando os 48 reservatórios estudados ao longo das 11 bacias e sub-bacias hidrográficas do Estado Ceará.

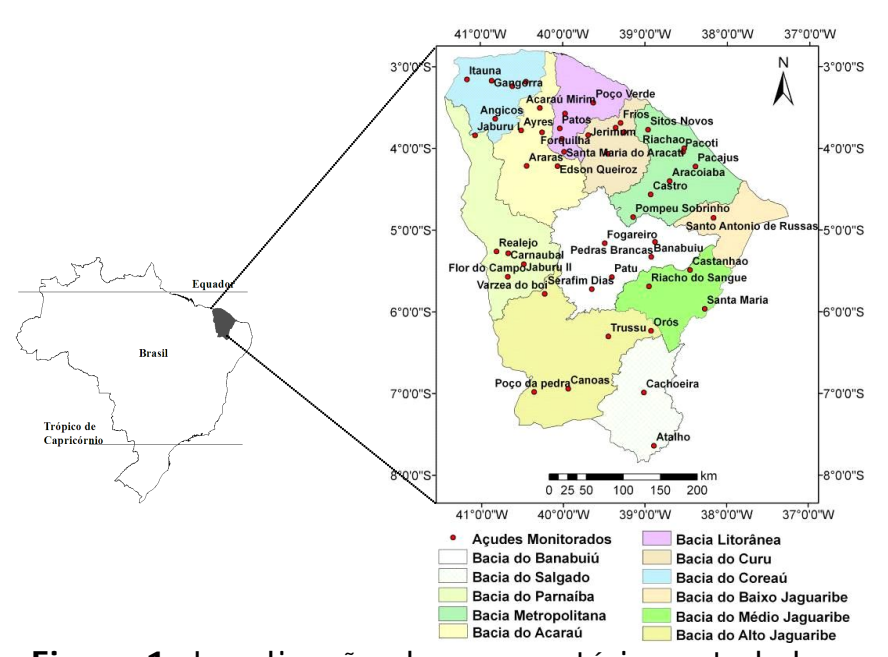

Figura 1. Localização dos reservatórios estudados, inseridos no Estado do Ceará

Localizado na zona do "Polígono das Secas", o Estado do Ceará é caracterizado por anos de baixos níveis pluviométricos anuais sendo necessária, para assegurar uma produção agrícola em épocas de estiagem, a prática de irrigação. O Ceará pertence a duas províncias hidrogeológicas: ao escudo oriental e à província costeira. Cerca de $70 \%$ da área do Ceará são compostos de embasamento cristalino, representando $21 \%$ do total do cristalino nordestino $\left(500.000 \mathrm{~km}^{2}\right)$, caracterizado por solos de pequena espessura $(\leq 2 \mathrm{~m})$. Há, também, bacias sedimentares, como as de Iguatu, do Apodi, do Araripe e da Ibiapaba. Além dos aquíferos existentes nestas conformações, existem áreas de aluviões e o sedimento costeiro, que é formado por sistemas dunas: paleodunas e formação barreiras (Silva et. al., 2007).

A região meridional e o centro-oriental do Ceará são drenados pelo rio Jaguaribe, o maior do Estado, que corre numa extensão de 800 kilômetros. Ao norte, destaca-se o rio 
Acaraú e a oeste, o rio Poti que, após atravessar o boqueirão existente na Chapada do Ibiapaba, se junta ao rio Parnaíba, já em território do Estado do Piauí. Encontram-se, ainda, entre os mais importantes do Estado, os rios Salgado, Conceição, Banabuiú, Trussu, Pacoti e Piranji (Silva et al., 2007).

Com exceção do trecho ao longo da costa e das chapadas e pequenas serras, o clima em boa parte do território do Estado do Ceará é semiárido, com médias pluviométricas inferiores a $600 \mathrm{~mm}$ e irregularidade nas precipitações, o que ocasiona secas periódicas. Em consequência deste fenômeno, os cursos d'água são temporários, permanecendo secos ao longo de todo o verão e a vegetação dominante é a da caatinga, com sua paisagem típica, de pequenas árvores retorcidas.

Os dados das concentrações químicas das águas dos açudes, para o período de 1998/2009, no total de 807 amostras utilizados foram provenientes do banco de dados da COGERH. Os parâmetros considerados no presente estudo foram: cloreto $\left(\mathrm{Cl}^{-}\right)$, cálcio $\left(\mathrm{Ca}^{2+}\right)$, magnésio $\left(\mathrm{Mg}^{2+}\right)$, sódio $\left(\mathrm{Na}^{+}\right)$, bicarbonato $\left(\mathrm{HCO}_{3}^{-}\right)$, condutividade elétrica (CE) e a razão de adsorção de sódio (RAS). Os açudes monitorados na área do estudo foram georreferenciados por intermédio de GPS de Navegação.

A semelhança na salinidade das águas entre os açudes do estado do Ceará foi estimada pelo emprego da técnica de análise multivariada, análise de agrupamento hierárquico (AAH), processada no software SPSS 16.0. As amostras de água foram agrupadas com base no seu grau de semelhança definida pela variação entre os coeficientes de agrupamento de dois grupos consecutivos, como descrito por Hair et al. (2005). Para se reduzir os erros devidos às escalas e as unidades das variáveis selecionadas, os dados foram normalizados $(\mathrm{x}=0, \sigma=1)$, pela seguinte relação (Eq. 1):

$$
Y_{i j}=\frac{X_{i j}-\overline{X_{i}}}{S_{i}}
$$

sendo:

$\mathrm{X}_{\mathrm{ij}}$ - o valor da j-ésima observação da i-ésima variável

$X_{i}$ - a média da variável $X_{i j}$

$\mathrm{S}_{\mathrm{i}}$ - o desvio padrão da variável $\mathrm{X}_{\mathrm{ij}}$

$\mathrm{Y}_{\mathrm{ij}}$ - a j-ésima observação da i-ésima variável transformada

Como as variáveis classificatórias adotadas neste estudo são variáveis reais e, portanto, mensuradas em uma escala de intervalo, adotou-se a combinação da distância Euclidiana ao quadrado para a formação da matriz de similaridade e o algoritmo do método de ligação Ward (método da variância mínima) (Hair et al., 2005; Palácio et al., 2009).

Os dados médios de cada parâmetro analisado dos respectivos grupos formados foram submetidos à análise de teste de média e confrontados pelo teste " $t$ " a nível de significância de 1\%, através do programa SPSS 16.0 for Windows.

A técnica de estatística multivariada Análise Fatorial/Análise de Componentes Principais (AF/ACP) foi aplicada a partir dos dados totais, independente dos grupos formados na $\mathrm{AAH}$, para identificar os fatores fundamentais que governam a salinidade das águas dos reservatórios do Ceará.

$\mathrm{Na}$ forma de matriz, os dados originais eram expressos por $\mathrm{X}=\left(\mathrm{x}_{\mathrm{i}, \mathrm{j}}\right)$, em que $\mathrm{i}=1 \ldots \mathrm{n}$ amostragens $(807) \mathrm{e} \mathrm{j}=1$...p variáveis (7). Na aplicação da técnica AF/ ACP, a primeira etapa é transformar a matriz de dados originais em uma matriz de correlação [R] (p x p), para p igual aos 7 parâmetros de qualidade de água analisados neste estudo. Após a definição da matriz de correlação realizou-se uma inspeção entre os parâmetros com o objetivo de identificar as variáveis mais específicas, visto que a finalidade da AF/ACP é obter componentes que ajudem a explicar referidas correlações.

A consistência geral dos dados foi aferida pelo método Kayser Mayer Olkim (KMO), (Monteiro \& Pinheiro, 2004; Andrade et al., 2007b). O número de componentes extraídos foi definido pelo critério da raiz latente, sendo considerados somente componentes com autovalor superior a um (Norusis, 1990).

A matriz dos componentes foi obtida na fase de extração; em seguida, foi feita a transformação ortogonal (rotação da matriz dos pesos fatoriais), gerando uma nova matriz pelo modelo de rotação ortogonal Varimax, adotado frequentemente em estudos de qualidade de água e processos hidrológicos (Helena et al., 2000; Palácio 2004; Andrade et al. 2007b).

\section{RESULTADOS E DISCUSSÃO}

\section{Análise de agrupamento hierárquico (AAH)}

A semelhança dos reservatórios do Estado do Ceará em relação à salinidade das águas, foi determinada pelo emprego da técnica de Análise Multivariada/Análise de Agrupamento Hierárquico (AAH). O dendrograma observado na Figura 2 evidencia que o ponto ótimo de corte da distância reescalonada de combinação se encontra entre 4,52 e 6,89, a qual é determinada através de interpolação pois, a partir desta, há um distanciamento maior na medida de similaridade para a formação de agrupamentos posteriores.

Para efeito de "corte" do dendrograma e assim se definir o número de grupos, optou-se por efetuá-lo na distância reescalonada de valor 5, dando origem a quatro grupos distintos. Resultados semelhantes foram encontrados por Palácio et al. (2009) utilizando AAH em águas superficiais da bacia do Curu, Ceará, onde foram formados quatro grupos homogêneos de similaridade das águas.

Verifica-se pela Figura 3 a distribuição no Estado dos grupos formados. O grupo 1 foi o de maior representatividade, com $68,75 \%$ do total de reservatórios estudados; este grupo foi formado por 33 reservatórios distribuídos praticamente por toda a região norte e parte do centro-leste do Estado; o grupo 2 foi composto por 12 reservatórios, o segundo mais representativo com $25 \%$ do total, todos os reservatórios da bacia do Alto Jaguaribe e Salgado, três da bacia do Parnaíba e dois da bacia do Acaraú; os últimos com grande representatividade mais ao sul e centro-oeste do Estado; já o grupo 3 foi constituído por 2 reservatórios, um da bacia Metropolitana e outro da bacia do Banabuiu; entretanto, o grupo 4 foi constituído exclusivamente por 1 açude localizado na bacia Metropolitana. 


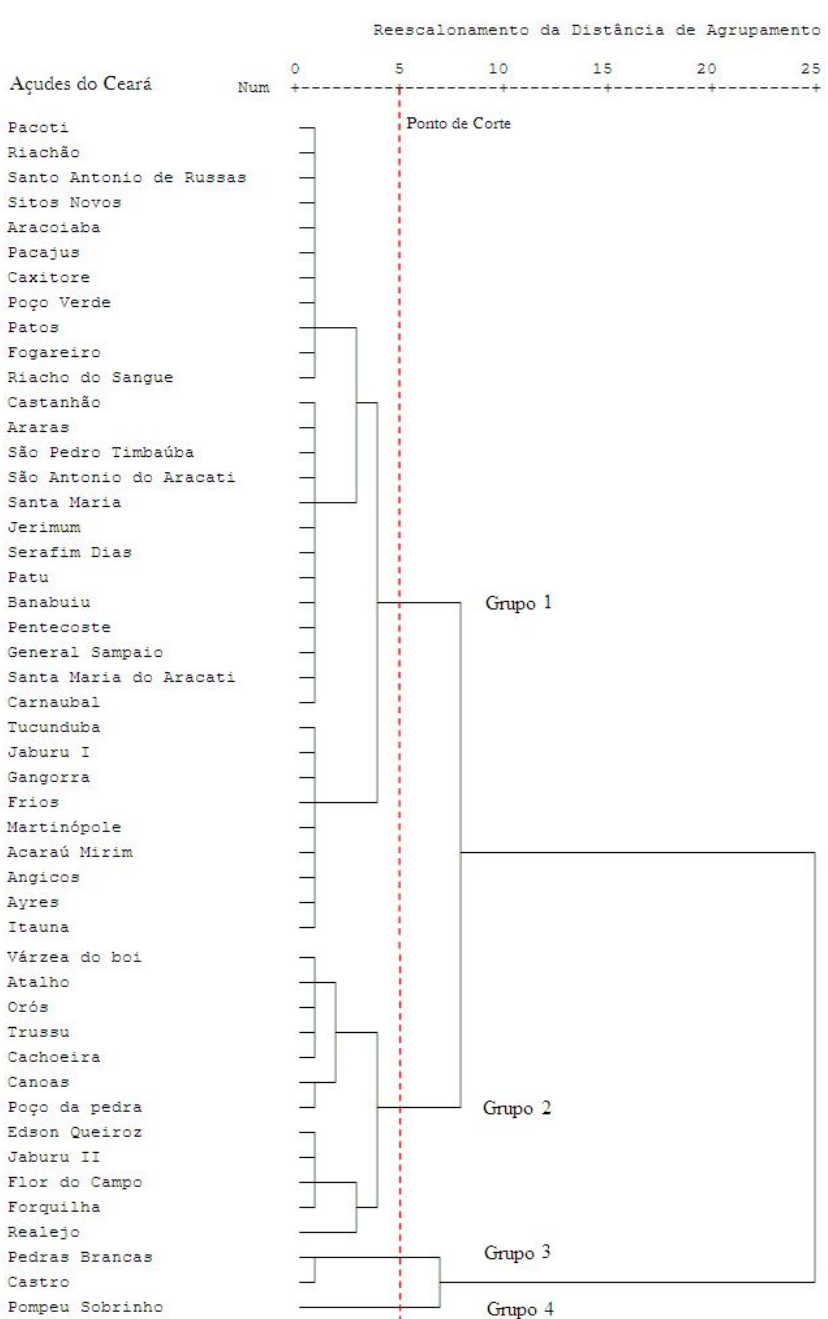

Figura 2. Dendrograma dos açudes do Ceará agrupados quanto à salinidade das águas

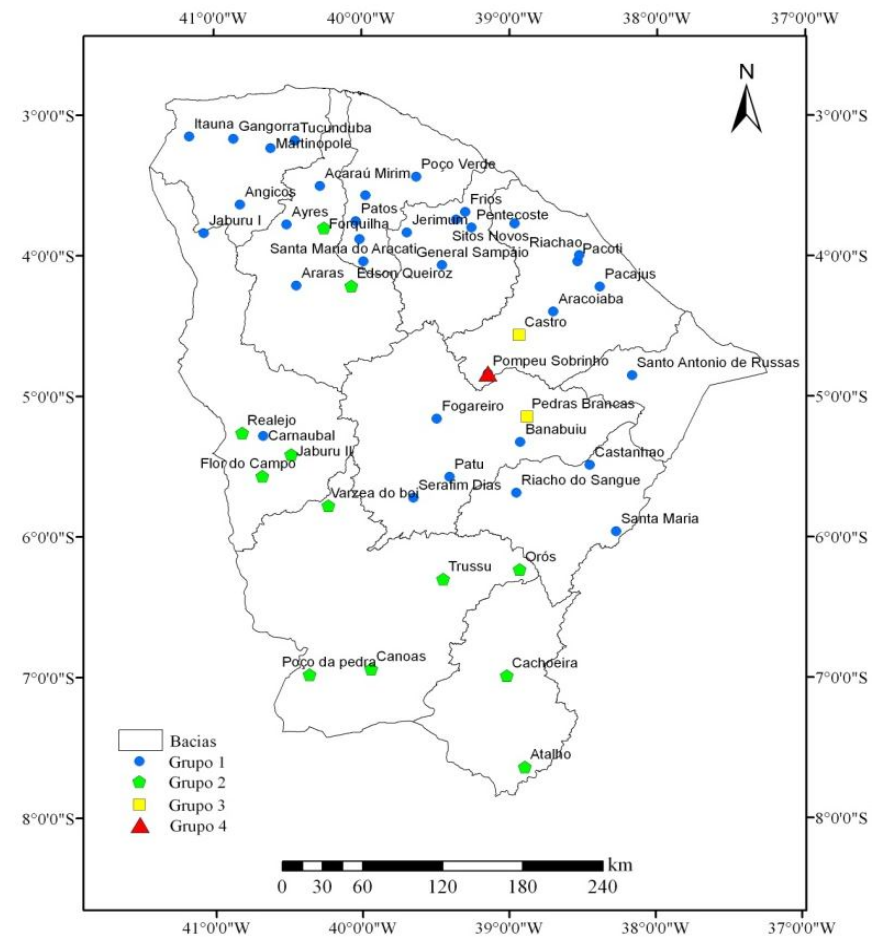

Figura 3. Distribuição espacial dos açudes do Ceará dentro dos agrupamentos formados pela técnica de estatística multivariada $\mathrm{AAH}$

A composição média, o desvio padrão e os valores máximos e mínimos dos atributos indicadores da salinidade das águas de cada grupo de açudes do Ceará, podem ser vistos na Tabela 1.

\section{Grupo 1}

Os atributos determinantes para a formação do grupo 1 foram $\mathrm{o} \mathrm{HCO}_{3}{ }^{-} \mathrm{e}^{\mathrm{o}} \mathrm{Ca}^{2+}$, que diferiram estatisticamente a nível de $1 \%$,

Tabela 1. Valores médios das variáveis em estudo para os grupos da salinidade das águas dos reservatórios do Ceará, definidos pela técnica da AAH

\begin{tabular}{|c|c|c|c|c|c|}
\hline & Estatística & Grupo 1 & Grupo 2 & Grupo 3 & Grupo 4 \\
\hline $\begin{array}{c}\mathrm{HCO}_{3} \\
\left(\mathrm{mmol}_{\mathrm{C}} \mathrm{L}^{-1}\right)\end{array}$ & $\begin{array}{c}\text { Média } \pm \text { Desvio Padrão } \\
\text { Mínimo } \\
\text { Máximo }\end{array}$ & $\begin{array}{c}1,32 \pm 0,46 a \\
0,20 \\
3,67\end{array}$ & $\begin{array}{c}2,20 \pm 1,22 b c \\
0,11 \\
7,12\end{array}$ & $\begin{array}{c}1,79 \pm 0,37 b \\
0,84 \\
2,92\end{array}$ & $\begin{array}{c}2,50 \pm 0,42 c \\
1,61 \\
3,10\end{array}$ \\
\hline $\begin{array}{c}\mathrm{Ca}^{2+} \\
\left(\mathrm{mmol}_{\mathrm{c}} \mathrm{L}^{-1}\right)\end{array}$ & $\begin{array}{c}\text { Média } \pm \text { Desvio Padrão } \\
\text { Mínimo } \\
\text { Máximo }\end{array}$ & $\begin{array}{c}0,93 \pm 0,42 a \\
0,13 \\
3,03\end{array}$ & $\begin{array}{c}2,02 \pm 1,49 b c \\
0,18 \\
7,75\end{array}$ & $\begin{array}{c}1,93 \pm 0,51 b \\
0,64 \\
3,18\end{array}$ & $\begin{array}{c}2,48 \pm 0,80 \mathrm{c} \\
1,78 \\
4,475\end{array}$ \\
\hline $\begin{array}{c}\mathrm{Cl}^{-} \\
\left(\mathrm{mmol}_{\mathrm{c}} \mathrm{L}^{-1}\right)\end{array}$ & $\begin{array}{c}\text { Média } \pm \text { Desvio Padrão } \\
\text { Mínimo } \\
\text { Máximo }\end{array}$ & $\begin{array}{c}3,06 \pm 1,79 a \\
0,26 \\
18,60\end{array}$ & $\begin{array}{c}1,79 \pm 1,42 \mathrm{a} \\
0,12 \\
7,78\end{array}$ & $\begin{array}{c}8,59 \pm 3,08 b \\
2,21 \\
17,52 \\
\end{array}$ & $\begin{array}{c}21,61 \pm 10,08 \mathrm{c} \\
8,23 \\
44,91 \\
\end{array}$ \\
\hline $\begin{array}{c}\mathrm{Mg}^{2+} \\
\left(\mathrm{mmol}_{\mathrm{c}} \mathrm{L}^{-1}\right)\end{array}$ & $\begin{array}{c}\text { Média } \pm \text { Desvio Padrão } \\
\text { Mínimo } \\
\text { Máximo }\end{array}$ & $\begin{array}{c}1,23 \pm 0,63 a \\
0,08 \\
3,31\end{array}$ & $\begin{array}{c}0,87 \pm 0,67 a \\
0,04 \\
3,68\end{array}$ & $\begin{array}{c}3,19 \pm 1,03 b \\
0,55 \\
6,30\end{array}$ & $\begin{array}{c}5,66 \pm 2,95 \mathrm{c} \\
2,57 \\
12,33\end{array}$ \\
\hline $\begin{array}{c}\mathrm{Na}^{+} \\
\left(\mathrm{mmol}_{\mathrm{c}} \mathrm{L}^{-1}\right)\end{array}$ & $\begin{array}{c}\text { Média } \pm \text { Desvio Padrão } \\
\text { Mínimo } \\
\text { Máximo }\end{array}$ & $\begin{array}{c}2,48 \pm 1,39 a \\
0,18 \\
17,11\end{array}$ & $\begin{array}{c}1,77 \pm 1,12 \mathrm{a} \\
0,12 \\
6,40\end{array}$ & $\begin{array}{c}5,86 \pm 2,81 b \\
0,99 \\
16,19\end{array}$ & $\begin{array}{c}10,47 \pm 5,00 \mathrm{c} \\
4,64 \\
21,79\end{array}$ \\
\hline $\begin{array}{c}C E \\
\left(d S \mathrm{~m}^{-1}\right)\end{array}$ & $\begin{array}{c}\text { Média } \pm \text { Desvio Padrão } \\
\text { Mínimo } \\
\text { Máximo }\end{array}$ & $\begin{array}{c}0,43 \pm 0,21 a \\
0,07 \\
1,71\end{array}$ & $\begin{array}{c}0,34 \pm 0,23 a \\
0,02 \\
1,64\end{array}$ & $\begin{array}{c}1,04 \pm 0,38 b \\
0,27 \\
2,52\end{array}$ & $\begin{array}{c}2,15 \pm 1,11 \mathrm{c} \\
0,69 \\
4,19\end{array}$ \\
\hline $\begin{array}{c}\text { RAS } \\
\left(\mathrm{mmol} \mathrm{L}^{-1}\right)^{0,5}\end{array}$ & $\begin{array}{c}\text { Média } \pm \text { Desvio Padrão } \\
\text { Mínimo } \\
\text { Máximo }\end{array}$ & $\begin{array}{c}2,45 \pm 1,45 a \\
0,19 \\
21,17\end{array}$ & $\begin{array}{c}1,80 \pm 1,13 a \\
0,10 \\
6,00\end{array}$ & $\begin{array}{c}3,68 \pm 1,62 b \\
1,58 \\
9,00\end{array}$ & $\begin{array}{c}5,16 \pm 1,66 c \\
2,56 \\
8,34\end{array}$ \\
\hline
\end{tabular}


dos demais grupos. Trata-se de um grupo formado por açudes que apresentaram as menores concentrações médias desses íons, com valores de 1,32 e 0,93 $\mathrm{mmol}_{\mathrm{c}} \mathrm{L}^{-1}$ para, respectivamente, $\mathrm{HCO}_{3}^{-} \mathrm{e} \mathrm{Ca}^{2+}$. Em ambos os íons, as menores concentrações devem estar associadas ao transporte conjunto na forma molecular de carbonato de cálcio $\left(\mathrm{CaCO}_{3}\right)$. Essas concentrações, por sua vez, não são preocupantes pois, de acordo com Ayers \& Westcot (1999), valores inferiores a 1,5 $\mathrm{mmol}_{c} \mathrm{~L}^{-1}$ para $\mathrm{HCO}_{3}^{-}$e $20 \mathrm{mmol}_{c} \mathrm{~L}^{-1}$ para $\mathrm{Ca}^{2+}$ expressam águas com baixo grau de restrição para uso na irrigação. Os referidos autores afirmam, ainda, que a função do cálcio no solo não está completamente estabelecida e, ao que parece, ele reduz os efeitos tóxicos dos íons $\mathrm{Na}^{+}$e $\mathrm{Mg}^{2+}$ quando são absorvidos pelas raízes. O cálcio também confere a dureza da água, podendo formar incrustações nas tubulações de irrigação (Arraes et al., 2009).

Outra característica deste grupo além dos menores valores dos íons $\mathrm{HCO}_{3}^{-}$e $\mathrm{Ca}^{2+}$, está ligada a valores mais elevados do $\mathrm{Cl}^{-}$e $\mathrm{Na}^{+}$em relação ao grupo 2. Para o grupo 1, a concentração média do íon $\mathrm{Cl}^{-}$apresentou valor de $3,06 \mathrm{mmol}_{\mathrm{c}} \mathrm{L}^{-1}$ superior ao limite de $3 \mathrm{mmol}_{\mathrm{c}} \mathrm{L}^{-1}$ permitido para uso na irrigação, de acordo com Ayers \& Westcot (1999). Para o íon $\mathrm{Na}^{+}$o valor médio de $2,48 \mathrm{mmol}_{\mathrm{c}} \mathrm{L}^{-1}$ apresentou-se inferior ao limite para irrigação de $3 \mathrm{mmol}_{\mathrm{c}} \mathrm{L}^{-1}$, no entanto, foram maiores do que as concentrações observadas no grupo 2 .

Esses valores mais elevados de $\mathrm{Cl}^{-}$e $\mathrm{Na}^{+}$do grupo $1 \mathrm{em}$ relação ao grupo 2, parecem estar ligados à localização dos reservatório mais ao norte do Estado (Figura 3). Essas águas tendem a apresentar maior concentração de $\mathrm{Cl}^{-} \mathrm{e} \mathrm{Na}^{+}$devido à proximidade com o litoral, recebendo influência dos aerossóis marinhos (Meireles et al., 2007). Já as águas dos reservatórios localizados na bacia do Banabuiu, mais ao centro-leste do Estado, sofrem influência das rochas cristalinas da região, com predominância de sódio e potássio na sua composição (Melo et al., 2003).

\section{Grupo 2}

O grupo 2 foi composto por reservatórios que apresentaram as menores salinidades das águas. Os indicadores $\mathrm{Cl}^{-}, \mathrm{Mg}^{2+}$, $\mathrm{Na}^{+}, \mathrm{CE}$ e RAS expuseram os menores valores médios observados em relação aos demais grupos (Tabela 1); no entanto, não diferiram estatisticamente a nível de $1 \%$, de significância do grupo 1; já os íons $\mathrm{HCO}_{3}^{-} \mathrm{e} \mathrm{Ca}^{2+}$ apresentaram a segunda maior concentração entre os grupos, assemelhandose estatisticamente ao nível de $1 \%$ aos grupos 3 e 4 . Apesar de nenhum parâmetro ter diferenciado estatisticamente esse grupo dos demais, fica evidente uma característica intermediária do grupo, apresentando características de baixas salinidades juntamente com o grupo 1 , e de maiores concentrações de $\mathrm{HCO}_{3}^{-} \mathrm{e} \mathrm{Ca}^{2+}$ junto com os grupos 3 e 4 .

Os menores valores médios de CE da água nesse grupo, permanecendo inferiores a $0,7 \mathrm{dS} \mathrm{m}^{-1}$, indicam baixos teores de sais dissolvidos, sem restrição de uso na irrigação e adequada para todas as espécies de rebanho, aves confinadas e consumo humano (Ayres \& Westcot, 1999; Brasil, 2005). A RAS também apresentou baixos riscos de causar problemas de infiltração nos solos, com valor médio de 1,80 inferior ao limite estabelecido (RAS < 3) para uso na irrigação.
Para este grupo, os valores de $\mathrm{HCO}_{3}^{-}$e $\mathrm{Ca}^{2+}$ só não foram superiores às concentrações observadas no grupo 4. Os valores observados para o $\mathrm{Ca}^{2+}$ podem ser atribuídos à presença desse elemento nas rochas em que os reservatórios estão situados, notadamente nas bacias do Alto Jaguaribe e do Salgado. Nessas bacias existe uma forte participação do cálcio na composição química que, provavelmente, irá prevalecer no período da evaporação. A região é constituída por unidades litoestatigráficas que são portadoras de rochas carbonáticas, merecendo destaque os corpos de rochas carbonáticas do Grupo Orós (Sousa \& Vidal, 2005).

De acordo com a concentração média do íon $\mathrm{HCO}_{3}{ }^{-}$de 2,20 $\mathrm{mmol}_{\mathrm{c}} \mathrm{L}^{-1}$, as águas desses reservatórios apresentaram moderados riscos de causar toxidez às plantas com valores superiores ao limite estabelecido para uso na irrigação de 1,5 mmol $\mathrm{L}^{-1}$; já o íon $\mathrm{Ca}^{2+}$ apresentou valor médio para esse grupo de 2,02 mmol $\mathrm{L}^{-1}$, abaixo do limite de $20 \mathrm{mmol}_{\mathrm{c}} \mathrm{L}^{-1}$ permitido para irrigação (Ayres \& Westcot, 1999). Ainda de acordo com os autores supracitados, quando são utilizadas águas para irrigação com elevados teores de bicarbonatos pode ocorrer precipitação de cálcio, na forma de carbonato de cálcio, facilitando um suposto processo de sodificação do solo.

\section{Grupo3}

O grupo 3 foi formado pela similaridade existente entre as águas dos açudes Castro e Pedra Branca localizados, respectivamente, nas bacias metropolitana e do rio Banabuiu. Os atributos: $\mathrm{Cl}^{-}, \mathrm{Mg}^{2+}, \mathrm{Na}^{+}, \mathrm{CE}$ e RAS desse grupo diferiram a um nível de $1 \%$ de significância dos demais. Este grupo apresentou concentrações médias dos íons $\mathrm{Cl}^{-}$e $\mathrm{Na}^{+}$de 8,59 e $5,86 \mathrm{mmol}_{\mathrm{c}} \mathrm{L}^{-1}$, respectivamente. De acordo com Ayers \& Westcot (1999), concentrações superiores a $3 \mathrm{mmol}_{\mathrm{c}} \mathrm{L}^{-1}$ para esses íons podem causar danos às culturas, reduzindo a produtividade. No entanto, para o íon $\mathrm{Mg}^{2+}$, referido grupo apresentou concentrações médias dentro dos limites permitidos para irrigação $\left(5 \mathrm{mmol}_{c} \mathrm{~L}^{-1}\right)$, conforme o autor supracitado.

Outra característica determinante do grupo 3 se liga aos valores da RAS e CE, os quais diferiram estatisticamente a nível de $1 \%$ de significância dos demais grupos, expressando sua dissimilaridade. A RAS é o parâmetro que melhor expressa o risco de sodicidade das águas empregadas na irrigação e a CE é o parâmetro utilizado para expressar a concentração de sais solúveis totais na água (Frota Júnior et al., 2007). Pela análise dos dados contidos na Tabela 1, observa-se que as águas que compõem este grupo apresentaram moderado risco em gerar problemas de infiltração (RAS > 3) e salinidade nos solos $\left(\mathrm{CE}<3 \mathrm{dS} \mathrm{\textrm {m } ^ { - 1 }}\right.$ ), razão porque podem ser usadas na irrigação apenas de solos de textura grossa (arenoso) sem perigo de atingir elevados níveis de sódio trocável, ou quando houver lixiviação moderada de sais.

Essas elevadas concentrações de sais verificadas para os reservatórios que compõem o grupo 3 , podem ser relacionadas à pressão da cidade de Itapiúna ao açude Castro, aliado às águas novas nas primeiras coletas, pois o reservatório foi concluído em 1997, pouco tempo antes do início do monitoramento. Para o açude Pedras Brancas, as elevadas concentrações dos sais devem estar associadas ao modo de operação do reservatório que, aliado à elevada taxa de 
evaporação potencial das águas no período de estiagem, promove a concentração dos sais.

\section{Grupo4}

Já para o grupo 4, constituído apenas do açude Pompeu Sobrinho, o fator determinante para a formação se constituiu dos maiores valores de todos os indicadores analisados nesse estudo; apenas os íons $\mathrm{HCO}_{3}^{-} \mathrm{e} \mathrm{Ca}^{2+}$, não diferiram estatisticamente ao nível de $1 \%$ de significância do grupo 2 , sendo que os demais elementos desse grupo se diferenciaram dos demais grupos. A característica desse reservatório o torna como o de maior problema de salinidade, diferencionando-o dos açudes estudados no Estado do Ceará. Este fato deve estar ligado à litologia predominante no Estado, e ao superdimensionamento do açude Pompeu Sobrinho, que ocasiona longos períodos sem renovação das águas por extravasamento do reservatório, cuja última sangria ocorreu em 1974 agravando, assim, o processo de acumulação de sais ao longo do tempo proporcionado pela evaporação.

Em estudos realizados pela FUNCEME (2002) entre os anos de 1999 a 2000, sobre a qualidade das águas em reservatórios superficiais da bacia metropolitana do Estado do Ceará, onde o reservatório Pompeu Sobrinho está localizado, tornou-se patente que as águas do reservatório apresentaram as maiores salinidades, com valor médio de $\mathrm{CE}$ de $5,5 \mathrm{dS} \mathrm{m}^{-1}$ para o período. De acordo com este estudo, a causa da salinização do Pompeu Sobrinho é resultado do superdimensionamento deste reservatório, que dificulta a renovação das águas.

O efeito da concentração dos sais causada pela evaporação dos reservatórios no período mais seco, é comum nas regiões semiáridas, nas quais chega, o déficit hídrico no período seco, a $858 \mathrm{~mm}$, sendo que este efeito se agrava com a falta de renovação das águas. Resultados semelhantes foram encontrados por Andrade et al. (2005) monitorando $22 \mathrm{~km}$ do trecho perenizado do rio Trussu na região Centro-Sul do Estado do Ceará, por Palácio et al. (2009), em estudos com as águas superficiais da bacia do Curu, Ceará.

As águas do grupo 4 detêm as maiores concentrações dos íons $\mathrm{Na}^{+}, \mathrm{Cl}^{-}$e $\mathrm{Mg}^{2+}$, apresentando de acordo com os limites estabelecidos por Ayers \& Westcot (1999) riscos severos de causar toxidez às plantas com o uso na irrigação. A concentração média do $\mathrm{Na}^{+}$, para esse grupo, foi de $10,47 \mathrm{mmol}_{\mathrm{c}}$ $\mathrm{L}^{-1}$, enquanto o $\mathrm{Cl}^{-}$apresentou concentração média de 21,61 mmol $\mathrm{L}^{-1}$ e o $\mathrm{Mg}^{2+}$ de 5,66 mmol $\mathrm{L}^{-1}$. Estas concentrações são extremamente preocupantes, inclusive superiores aos limites aceitáveis para consumo humano, de acordo com a Portaria 518/05 do Ministério da Saúde (Brasil, 2004), pois o alto consumo de sódio é utilizado, atualmente, como indicador na predição de doenças cardiovasculares (Molina et al., 2003). Em referência à quantidade usualmente encontrada em águas doces, os cloretos não são prejudiciais; entretanto, altas concentrações do íon cloreto podem trazer restrições ao sabor e, desta maneira, levar o consumidor a procurar outras fontes de suprimento, frequentemente de qualidade sanitária pouco desejável (Lemos et al., 2010).

Constatou-se, ainda, que as águas do grupo 4 apresentaram a maior média de condutividade elétrica, $2,15 \mathrm{dS} \mathrm{m}^{-1}$, resultado condizente com os dados mais elevados dos componentes iônicos $\left(\mathrm{Na}^{+}, \mathrm{Ca}^{2+}, \mathrm{Mg}^{2+}, \mathrm{Cl}^{-}\right.$e $\left.\mathrm{HCO}_{3}^{-}\right)$. Os valores da $\mathrm{CE}$ registrados nesse grupo podem promover diarréia temporária em bovinos e excrementos aquosos nas aves. Quanto à irrigação, ela só pode ser utilizada, mesmo em solos com drenagem adequada, adotando-se práticas especiais de controle da salinidade, devendo, portanto, ser utilizada na irrigação de espécies vegetais de alta tolerância aos sais (Ayres \& Westcot, 1999).

\section{Análise de componentes principais (ACP)}

Para identificação dos parâmetros mais significativos na variabilidade da salinidade dos reservatórios do Ceará, aplicouse a técnica de estatística multivariada Análise Fatorial/Análise de Componentes Principais (AF/ACP). Na primeira etapa foram selecionadas as variáveis $\mathrm{Ca}^{2+}, \mathrm{Cl}^{-}, \mathrm{Mg}^{2+}, \mathrm{Na}^{+}, \mathrm{CE}$ e $\mathrm{HCO}_{3}^{-}$, excluindo-se apenas a RAS atingindo, no teste de adequacidade Kaiser-Meyer-Olkin (KMO), um índice igual a 0,833 (ótimo), demonstrando que o modelo promoverá redução significante na dimensão dos dados originais, conforme orientam Souza \& Tundisi (2000), Palácio (2004) e Hair et al. (2005).

A matriz de correlação composta pelas variáveis que se adequaram pode ser vista na Tabela 2 . Pela referida matriz, observa-se que as variáveis estudadas, com exceção do $\mathrm{Ca}^{2+}$, apresentaram correlação superior a 0,5 com pelo menos três outras variáveis. Helena et al. (2000) e Andrade et al. (2007a) consideram que coeficientes de correlação superiores a 0,5 expressam uma forte relação. Pode-se observar também, que a

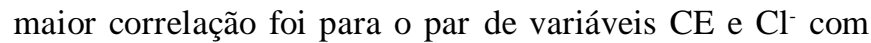
coeficiente de correlação superior a 0,9. Altas correlações entre a $\mathrm{CE}$ e o $\mathrm{Cl}^{-}$também foram constatadas em estudos realizados por Girão et al. (2007), no rio Jaibaras, Ceará, e Andrade et al. (2007a) na bacia do rio Acaraú, Ceará. Essa correlação era esperada, uma vez que a CE expressa os sais presentes na água e, durante a estação seca, ocorre o aumento do cloreto nas águas dos açudes, devido à alta taxa de evaporação no semiárido cearense. Acredita-se que, além das condições naturais da região, proximidade do mar e litologia, as concentrações desses elementos também sejam decorrentes de ações antrópicas, como resíduos de esgotos domésticos (Palácio, 2004).

Tabela 2. M atriz de correlação das variáveis analisadas nas águas dos açudes do Ceará

\begin{tabular}{|c|c|c|c|c|c|c|}
\hline & $\mathrm{Cl}^{-}$ & $\mathrm{Mg}^{+2}$ & $\mathrm{Na}^{+}$ & CE & $\mathrm{Ca}^{+2}$ & $\mathrm{HCO}_{3}$ \\
\hline $\begin{array}{c}\mathrm{Cl}^{-} \\
\mathrm{Mg}^{+2}\end{array}$ & $\begin{array}{c}1 \\
0,831\end{array}$ & 1 & & & & \\
\hline $\mathrm{Na}^{+}$ & 0,883 & $\underline{0,711}$ & 1 & & & \\
\hline $\mathrm{CE}$ & $\overline{0,916}$ & 0,839 & 0,859 & 1 & & \\
\hline $\mathrm{Ca}^{+2}$ & $\overline{0,418}$ & $\overline{0,367}$ & $\overline{0,390}$ & 0,454 & 1 & \\
\hline $\mathrm{HCO}_{3}{ }^{-}$ & 0,281 & 0,260 & 0,335 & 0,342 & $\underline{0,505}$ & 1 \\
\hline
\end{tabular}

Na etapa seguinte, quando da extração dos fatores, o modelo que melhor se ajustou aos dados foi aquele composto por dois fatores com as raízes características superiores à unidade. Esses fatores comuns e independentes (componente principal) reduzem a dimensão de variáveis interrelacionadas em dimensões menores para explicar a variabilidade dos dados 
originais (Andrade et al., 2007a). Para suplantar as dificuldades na identificação das variáveis mais significativas na matriz de pesos fatoriais, em decorrência de valores muito próximos entre si, aplicou-se a transformação ortogonal pelo emprego do algoritmo varimax, para a maximização e minimização dos maiores e menores autovalores, respectivamente. Pesquisadores como Helena et al. (2000), Silva \& Sacomani (2001) e Palácio (2004), obtiveram uma matriz de mais fácil interpretação com a aplicação do algoritmo varimax na elaboração da matriz transformada.

Os pesos fatoriais atribuídos a cada componente, as comunalidades de cada variável e a variância explicada após a aplicação do algoritmo podem ser observados na Tabela 3. Verifica-se que o primeiro componente (CP1) e o segundo componente (CP2) explicaram, respectivamente, 57,28 e 26,77\% da variância total dos dados, concentrando, em duas dimensões, 84,04\% das informações antes observadas em sete dimensões. Resultados semelhantes em termos de variância explicada foram encontrados por Toledo \& Nicolella (2002), Mendiguchía, et al. (2004) e Palácio (2004), trabalhando com águas superficiais.

Tabela 3. Valores das comunalidades e pesos fatoriais dos componentes das matrizes na extração, após a rotação pelo al goritmo varimax

\begin{tabular}{ccrc}
\hline Variáveis & Comunalidades & \multicolumn{1}{c}{ CP1 } & CP2 \\
$\mathrm{Cl}^{-}$ & 0,939 & $\underline{0,950}$ & 0,191 \\
$\mathrm{Mg}^{+2}$ & 0,813 & $\underline{0,888}$ & 0,155 \\
$\mathrm{Na}^{+}$ & 0,843 & $\underline{0,889}$ & 0,230 \\
$\mathrm{CE}$ & 0,930 & $\underline{0,929}$ & 0,258 \\
$\mathrm{Ca}^{+2}$ & 0,723 & 0,277 & $\underline{0,804}$ \\
$\mathrm{HCO}_{3}{ }^{-}$ & 0,794 & 0,119 & $\underline{0,883}$ \\
Autovalor & & 3,44 & 1,61 \\
Variância Explicada \% & & 57,28 & 26,77 \\
Variância Acumulada \% & & 57,28 & 84,04 \\
\hline
\end{tabular}

Verifica-se ainda, na Tabela supracitada, os pesos fatoriais dos componentes um e dois (CP1; CP2). Observa-se que no $\mathrm{CP} 1$ apenas as variáveis $\mathrm{Ca}^{2+}$ e $\mathrm{HCO}_{3}^{-}$apresentaram peso fatorial menor do que 0,300 , sugerindo que os demais parâmetros $\mathrm{Cl}^{-}, \mathrm{CE}, \mathrm{Na}^{+}$e $\mathrm{Mg}^{2+}$ são os mais significativos na definição e na variabilidade da salinidade das águas do Estado do Ceará; já para o $\mathrm{CP} 2$, as variáveis $\mathrm{Ca}^{2+}$ e $\mathrm{HCO}_{3}^{-}$apresentaram peso maior que 0,800 , sugerindo ser essas as únicas variáveis significativas neste componente, pois quanto mais elevado for o peso fatorial em módulo, maior a interrelação da variável com o fator.

Através dos valores apresentados pelas comunalidades das variáveis (Tabela 3), observa-se que os quatro parâmetros explicados pelo CP1 tiveram valores de comunalidade superiores a 0,888 e os parâmetros explicados pelo CP2 apresentaram valores superiores a 0,804 , sugerindo que o modelo aplicado sobre os dados explica melhor a variância dos parâmetros relacionados aos sais totais dissolvidos nas águas

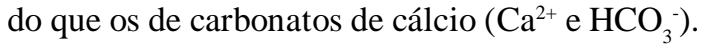

O CP1 apresentou maior associação com os sais totais dissolvidos nas águas. Por analisar, em uma escala estadual, esses elementos, podem ser oriundos tanto de fontes naturais (mineralização e aerossóis marinhos), como de fontes não naturais (esgotos domésticos) dependendo da região do Estado em que o reservatório está localizado, pois pesquisadores como Andrade et al. (2007b), estudando as águas da bacia do Acaraú, constataram que a origem comum desses minerais foi o intemperismo das rochas e consequente escoamento superficial das áreas drenadas; já Girão at al. (2007), estudando o rio Jaibaras, relacionaram os sais com dejetos de balneários e esgotos domésticos, enquanto Palácio (2004), em estudos na bacia do Rio Trussu, identificou, como fonte de cloreto e sódio, as lavagens de roupa e os esgotos domésticos.

$\mathrm{O} C \mathrm{P} 2$ recebeu maior peso para os parâmetros $\mathrm{Ca}^{2+} \mathrm{HCO}_{3}^{-}$; este componente pode ser associado à transferência do íon $\mathrm{Ca}^{2+}$ do sedimento para água, na forma de $\mathrm{CaCO}_{3}$. Os resultados estão de acordo com os encontrados por Santos et al. (2004), avaliando o processo de acumulação de sais na bacia do rio Contas, Bahia, que observou uma correlação maior entre os elementos $\mathrm{Ca}^{2+} \mathrm{e} \mathrm{CO}_{3}^{-}$, em decorrência do transporte desses elementos na forma molecular $\mathrm{CaCO}_{3}$.

\section{CONCLUSÕES}

1. A AAH formou quatro grupos distintos, sendo que nos grupos 1 e 2 a salinidade das água é definida pelas condições naturais. O grupo 1, influenciado pela composição química das rochas, presença dos aerossóis marinhos e alta evaporação; o grupo 2, pela composição química das rochas do cristalino e presença de rochas calcárias; os grupos 3 e 4, além das condições naturais, sofrem acréscimo na salinidade em função das atividades antrópicas exercidas nas proximidades dos reservatórios. O reservatório que forma o grupo 4 apresenta um agravante a mais, ou seja, o superdimensionamento do reservatório, que dificulta a renovação de suas águas.

2. O emprego da AF/ACP promoveu a redução de sete características das águas superficiais do Estado do Ceará para dois componentes, que explicam 84,04\% da variância total, mostrando que os parâmetros mais representativos na variabilidade da salinidade das águas foram $\mathrm{Cl}, \mathrm{CE}, \mathrm{Na}^{+} \mathrm{e} \mathrm{Mg}^{2+}$, relacionados com a solubilidade dos sais (naturais e/ou não naturais).

\section{AgradeCIMENTos}

Os autores agradecem à Companhia de Gestão de Recursos Hídricos do Ceará (COGERH), pela disponibilização do seu banco de dados utilizado neste trabalho.

\section{LITERATURA CITADA}

Andrade, E. M.; Araújo, L. F. P.; Rosa, M. F.; Gomes. R. B.; Lobato, F. A. O. Fatores determinantes da qualidade das águas superficiais na bacia do Alto Acaraú, Ceará, Brasil. Ciência Rural, v.37, p.1791-1797, 2007a.

Andrade, E. M.; Araújo, L. F. P.; Rosa, M. F.; Paulino, W. D.; Alves, A. B. Seleção dos indicadores da qualidade das águas superficiais pelo emprego da análise multivariada. Engenharia Agrícola, v.27, p.683-690, 2007b. 
Andrade, E. M.; Palácio, H. A. Q.; Crisóstomo, L. A.; Souza, I. H.; Teixeira, A. S. Índice de qualidade de água, uma proposta para o vale do rio Trussu, Ceará. Revista Ciência Agronômica, v.36, p.135-142, 2005.

Arraes, F. D. D.; Andrade, E. M.; Palácio, H. A. Q.; Frota, J. I. J.; Santos, J. C. N. Identificação dos íons determinantes da condutividade elétrica nas águas superficiais da Bacia do Curu, Ceará. Revista Ciência Agronômica, v.40, p.346-355, 2009.

Ayers, R. S.; Westcot, D. W. A qualidade da água na agricultura. 2.ed. Campina Grande: UFPB, 1999. 153p. FAO, Irrigação e Drenagem, 29

Brasil. Ministério da Saúde. Portaria n.518, de 25 de março de 2004. "Estabelece os procedimentos e responsabilidades relativos ao controle e vigilância da qualidade da água para consumo humano e seu padrão de potabilidade, e dá outras providências". Diário Oficial da República Federativa do Brasil. Brasília, 26 de março de 2004. seção 1.

Brasil. Ministério do Meio Ambiente. Resolução CONAMA N ${ }^{\circ}$ 357 de 17 de março de 2005. Dispõe sobre classificação dos corpos de água e diretrizes ambientais para o seu enquadramento, bem como estabelece as condições e padrões de lançamento de efluentes, e dá outras providências. <http://www.mma.gov.br/conama/res/res05/res35705.pdf. 11 Set. 2010.

Frota Júnior, J. I.; Andrade, E. M.; Meireles, A. C. M.; Bezerra, A. M.; Souza, B. F. S. Influência antrópica na adição de sais no trecho perenizado da bacia hidrográfica do Curu, Ceará. Revista Ciência Agronômica, v.38, p.142-148, 2007.

FUNCEME - Fundação Cearense de Meteorologia e Recursos Hídricos. Projeto estudo da qualidade das águas em reservatórios superficiais da bacia Metropolitana. Fortaleza: FUNCEME, v.1, 2002. 80p.

Girão, E. G.; Andrade, E. M.; Rosa, M. F.; Araújo, L. F. P.; Meireles, A. C. M. Seleção dos indicadores da qualidade de água no Rio Jaibaras pelo emprego da análise da componente principal. Revista Ciência Agronômica, v.38, p.17-24, 2007.

Hair, J. F. J.; Anderson, R. E.; Tatham, R. L.; Black, W. C. Análise multivariada de dados. Trad. Santanna, A. S.; Chaves Neto, A. Porto Alegre: Bookman, 2005. 593p.

Helena, B.; Pardo, R.; Vega, M.; Barrado, E.; Fernandez J. M.; Fernandez, L. Temporal evolution of groundwater composition in an alluvial aquifer (Pisuerga river, Spain) by principal component analysis. Water Research, v.34, p.807816, 2000.

Lemos, M.; Ferreira Neto, M.; Dias, N. da S. Sazonalidade e variabilidade espacial da qualidade da água na Lagoa do Apodi, RN. Revista Brasileira de Engenharia Agrícola e Ambiental, v.14, p.155-164, 2010.

Meireles, A. C. M.; Frischkorn, H.; Andrade, E. M. Sazonalidade da qualidade das águas do açude Edson Queiroz, Bacia do Acaraú, no Semiárido cearense. Revista Ciência Agronômica, v.38, p.25-31, 2007.
Melo, G. J.; Costa, C. E. F. S.; Cabral Neto, I. Avaliação hidroquímica e da qualidade das águas de um trecho do rio Açu, Rio Grande do Norte. Revista de Geologia, v.16, p.27-37, 2003.

Mendiguchía, C.; Moreno, C.; Galindo-Riâno, M. D.; GarcíaVarga, M. Using chemometric tools to assess anthropogenic effects in river water. A case study: Guadalquivir river (Spain). Analytica Chímica Acta, v.515, p.143-149, 2004.

Molina, M. D. B.; Cunha, R. S.; Herkenhoff, L. F.; Mill, J. G. Hipertensão arterial e consumo de sal em população urbana. Revista de Saúde Pública, v.37, p.743-750, 2003.

Monteiro, V. P.; Pinheiro, J. C. V. Critério para implantação de tecnologias de suprimentos de água potável em municípios cearenses afetados pelo alto teor de sal. Revista de Economia e Sociologia Rural, v.42, p.365-387, 2004.

Norusis, M. J. SPSS Base System User's Guide. Chicago: SPSS Inc, $1990.520 \mathrm{p}$.

Palácio, H. A. Q. Índice de qualidade das águas na parte baixa da bacia hidrográfica do rio Trussu, Ceará. Fortaleza: Universidade Federal do Ceará, 2004. 96p. Dissertação Mestrado

Palácio, H. A. Q.; Andrade, E. M.; Lopes, F. B.; Alexandre, D. M. B. Similaridade da qualidade das águas superficiais da bacia do Curu, Ceará, usando análise multivariada. Ciência Rural, v.39, p.2494-2500, 2009.

Santos, J. S.; Oliveira, E.; Bruns, R. E.; Gennari, R. F. Evaluation of the salt accumulation process during inundation in water resource of Contas river basin (Bahia-Brazil) applying principal component analysis. Water Research, v.38, p.1579-1585, 2004.

Silva, A. M. M.; Sacomani, L. B. Using chemical and physical parameters to define the quality of Pardo river water (Botucatu- SP-Brasil). Water Research, v.35, p.1609- 1616, 2001.

Silva, F. J. A.; Araújo, A. L.; Souza, R. O. Águas subterrâneas no Ceará - poços instalados e salinidade. Revista Tecnologia, v.28, p.136-159, 2007.

Silva Júnior, J. N; Sousa, A. R.; Sá, V. A. L.; Lima, B. P. Relações entre a concentração de íons e a salinidade de águas subterrâneas e superficiais visando à irrigação no sertão de Pernambuco. Revista Brasileira de Engenharia Agrícola e Ambiental, v.4, p.189-193, 2000.

Sousa, J. F.; Vidal, F. W. H. Rochas carbonáticas. Rio de Janeiro: CETEM, p.49-66, 2005.

Souza, A. D. G.; Tundisi, J. G. Hidrogeochemical comparative study of the Jaú and Jacaré-Guaçu river watersheds, São Paulo, Brazil. Revista Brasileira de Biologia, v.60, p. 63$570,2000$.

Toledo, L. G.; Nicolella, G. Índice de qualidade de água em microbacia sob uso agrícola e urbano. Scientia Agrícola, v.59, p.181-186, 2002. 